International Electronic Journal of Geometry

Volume 8 No. 2 PP. 1-8 (2015) @CIEJG

\title{
ELASTIC CURVES IN A TWO-DIMENSIONAL LIGHTLIKE CONE
}

\author{
GÖZDE ÖZKAN TÜKEL AND AHMET YÜCESAN
}

(Communicated by Kazım İLARSLAN)

\begin{abstract}
We establish the variational problem giving elastic curves in a twodimensional lightlike cone. The solutions of the variational problem produce two Euler-Lagrange equations. By using some technical of differential geometry, we solve the equations. Then, we classify elastic curves in the lightlike cone. Finally, we plot some of elastic curves in the lightlike cone.
\end{abstract}

\section{INTRODUCTION}

An elastic curve (or elastica) which is one of the most classical topics in the calculus of variations in a space, a surface or a manifold is defined as the curve satisfying a variational condition appropriate for interpolation problems. According to D. Bernouilli's idealization, all kinds of elastic curves minimize the total squared curvature $\int_{0}^{\ell} \kappa^{2}(s) d s$ among curves of the same length $\ell$ and first order boundary data [1]. For example, geodesics on a plane or a sphere and eight curve in Euclidean space are obvious examples of elastic curves (see for example $[3,4,6,13$,$] etc.).$

Like many of the problems explored by mathematicians of this era, the formal investigation of the elastica was motivated by a physical situation. The Euler-Bernoulli treatment of the elastica transformed a physics problem into one in mathematics. Examining the bending energy of a physical elastic rod was replaced by investigating the total squared curvature of a regular curve [14]. The total squared curvature functional has emerged as a useful quantity in the study of geodesics and the closed thin elastic rod is often used as a model for the DNA molecule [5]. So far, many authors have written several papers on elastic curves in Euclidean and non-Euclidean spaces. D. Singer obtained Euler-Lagrange equations of the elastic curves in three-dimensional Euclidean space and solved the equations by using Jacobi elliptic functions. He also generalized variational problem of elastic curves

Date: Received: July 9, 2015 and Accepted: August 12, 2015.

2010 Mathematics Subject Classification. 53A35, 53B30, 35A15, 74B20.

Key words and phrases. Elastic curves, Euler-Lagrange equations, Lightlike cone.

This work was supported by the Unit of Scientific Research Projects Coordination of Suleyman Demirel University under project 3356-D2-12. (Corresponding Author: Ahmet YÜCESAN). 
in Riemannian manifold of constant sectional curvature [13]. In [6], the authors examined the closed elastic curves in the manifolds with constant sectional curvature. On the other hand, G. Brunnet and P.E. Crouch gave differential equations for the main invariants of spherical elastic curves. They solved the equations by using Jacobi elliptic functions and presented a classification of the fundamental forms of elastic curves [4].

By using the same formulization, differential equation systems for elastic curves is studied and improved by many geometers in non-Euclidean spaces. In this paper, we give a special attention to Minkowski geometry which is a non-Euclidean space. In spite of the fact that Minkowski geometry and Euclidean geometry have a certain parallelism, in Minkowski geometry one can generate different results from Euclidean geometry for curves or surface due to its metric structure. Moreover Minkowski geometry is more complex than Euclidean geometry because Minkowski geometry includes both spherical geometry and hyperbolic geometry while Euclidean geometry includes only spherical geometry. Furthermore, there are three types curves-called as spacelike, timelike and lightlike-and two types surfaces-called as "degenerate" and "non-degenerate" - in Minkowski space while its Euclidean partner has a type curve and surface. For example, two-dimensional de Sitter space (Figure 1a) and two-dimensional hyperbolic space (Figure 1b) in three-dimensional Minkowski space $E_{1}^{3}$ corresponding a sphere in three-dimensional Euclidean space $E^{3}$ are non-degenerate surfaces and de Sitter two-space has timelike, spacelike and lightlike curves while hyperbolic two-space has only spacelike curves $[9,10]$. On the other part, two-dimensional lightlike cone (Figure 1c) which contains all of lightlike (null) vectors in three-dimensional Minkowski space $E_{1}^{3}$ is a degenerate surface and the lightlike cone has only spacelike curves [7, 9].

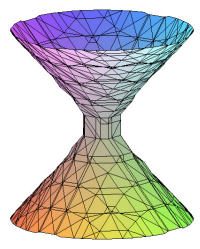

(a)

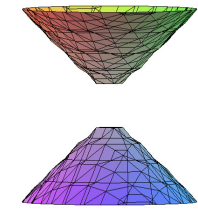

(b)

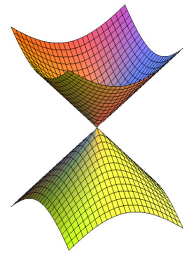

(c)

Figure 1. (a) De Sitter Space, (b) Hyperbolic Space, (c) Lightlike Cone in $E_{1}^{3}$.

It seems very interesting to work elastic curves on the hyperquadrics and the lightlike cone in three- dimensional Minkowski space $E_{1}^{3}$. Recently, Yücesan and Oral worked elastic curves on hyperquadrics in three-dimensional Minkowski space $E_{1}^{3}[11,16]$. However, elastic curves in a degenerate surface like a lightlike cone have never handled up till now. But curves in a lightlike cone introduced by $\mathrm{H}$. Liu. He gave some basic information to reader about structure and frame of the curves in the lightlike cone, then he characterized some curves in two and three-dimensional 
lightlike cone [7]. In [8] the authors also examined the properties and structures of cone curves two and three-dimensional lightlike cone by means of the representation formulas for spacelike curves in these lightlike cones. On the other hand, a problem of elastic curves in a two-dimensional lightlike cone arise as an open problem in Ph.D. thesis work of the first author [12]. According to the thesis work, solutions of this problem enable a nice connection between elastic curves on a two-dimensional lightlike cone and elastic strips with null directrix and pseudo-null directrix in three-dimensional Minkowski space $E_{1}^{3}$ [12]. In this paper, we do not handle to this topic, but we completely determine elastic curves in two-dimensional lightlike cone which directly contribute solution of the problem.

We firstly determine the variational problem giving elastic curve on a two- dimensional lightlike cone as a critical point of the functional $\int_{0}^{\ell}(-2 \kappa+\sigma) d s$ among curves with fixed initial points, directions and length $\ell$, where $\sigma$ is tension parameter. By using calculus of variations, we find out two Euler-Lagrange equations for elastic curves on the lightlike cone. Solving the equations by means of some techniques of differential geometry, we give explicit solutions of equilibrium equations of elastic curves with regard to the tension parameter and classify the solutions in the lightlike cone. Then, we plot these curves.

\section{Preliminaries}

Consider three-dimensional Minkowski space $E_{1}^{3}$ with the metric

$$
<x, y>=x_{1} y_{1}+x_{2} y_{2}-x_{3} y_{3}
$$

where $x=\left(x_{1}, x_{2}, x_{3}\right), y=\left(y_{1}, y_{2}, y_{3}\right) \in E_{1}^{3}$. Two-dimensional lightlike cone is defined as the set of all lightlike vectors of $E_{1}^{3}$

$$
\mathbb{Q}^{2}=\mathbb{Q}_{1}^{2}(0)=\left\{p \in E_{1}^{3} \mid<p, p>=0\right\}-\{(0,0,0)\}
$$

$[9,10]$. We usually use the Frenet frame when we study elastic curves in Euclidean or Minkowski space. However, the Frenet frame sometimes gives no information about the curve (see for an example [8]). Hence, we use the asymptotic orthonormal frame field introduced by Liu [7].

Assume that the curve given by

$$
\begin{aligned}
\gamma: I \subset \mathbb{R} & \rightarrow \mathbb{Q}^{2} \subset E_{1}^{3} \\
t & \rightarrow \gamma(t)
\end{aligned}
$$

is a regular curve in the two-dimensional lightlike cone $\mathbb{Q}^{2}$. Since $\langle\gamma, \gamma\rangle=0$ and $<\frac{d \gamma}{d t}, \gamma>=0, \gamma$ is a spacelike curve. This implies that curves in two-dimensional lightlike cone are spacelike. On the other hand, the arc length $s$ of the curve $\gamma(t)$ can be defined by

$$
d s^{2}=<d \gamma(t), d \gamma(t)>\text {. }
$$

If we take the arc length $s$ of the curve $\gamma(t)$ as the parameter and denote $\gamma(s)=$ $\gamma(t(s))$, then we have the spacelike unit tangent vector field of $\gamma(s)=\left(\gamma_{1}(s), \gamma_{2}(s), \gamma_{3}(s)\right)$ as the follow

$$
\gamma^{\prime}(s)=\frac{d \gamma(s)}{d s}=T(s)=\left(t_{1}(s), t_{2}(s), t_{3}(s)\right) .
$$


Thus, we choose the normal vector field $N=\left(n_{1}(s), n_{2}(s), n_{3}(s)\right)$ satisfying the following conditions:

$$
\begin{aligned}
& <T(s), T(s)>=<\gamma(s), N(s)>=1 \\
& <\gamma(s), \gamma(s)>=<N(s), N(s)>=<T(s), \gamma(s)>=0 .
\end{aligned}
$$

So, the frame field $\{\gamma, T, N\}$ constitutes an asymptotic orthonormal frame field in three-dimensional Minkowski space $E_{1}^{3}$ along the curve $\gamma$ in two-dimensional lightlike cone $\mathbb{Q}^{2}$ with the following derivative equations

$$
\begin{aligned}
& \gamma^{\prime}(s)=T(s), \\
& T^{\prime}(s)=\kappa(s) \gamma(s)-N(s), \\
& N^{\prime}(s)=-\kappa(s) T(s)
\end{aligned}
$$

where $\kappa$ is the cone curvature function.

For an arbitrary parameter $t$ of the curve $\gamma(t)$, the cone curvature function $\kappa$ is given by

$$
\kappa(t)=\frac{<\frac{d \gamma}{d t}, \frac{d^{2} \gamma}{d t^{2}}>^{2}-<\frac{d^{2} \gamma}{d t^{2}}, \frac{d^{2} \gamma}{d t^{2}}><\frac{d \gamma}{d t}, \frac{d \gamma}{d t}>}{2<\frac{d \gamma}{d t}, \frac{d \gamma}{d t}>^{5}}
$$

[7].

\section{Elastic Curves in a Two-Dimensional Lightlike Cone}

H. Liu studied curves in the lightlike cone and characterized some curves in the two-dimensional and three-dimensional lightlike cones [7]. In this section, we examine elastic curves in the lightlike cone $\mathbb{Q}^{2}$. An elastic curve minimizes the functional

$$
\int_{0}^{\ell}\left(<\gamma^{\prime \prime}, \gamma^{\prime \prime}>+\sigma<\gamma^{\prime}, \gamma^{\prime}>\right) d s
$$

with fixed length $\ell$ and boundary conditions in $\mathbb{Q}^{2}$. It is called a free elastica if the tension parameter $\sigma=0$ in this functional. From this point of view and by using derivative equations of the asymptotic orthonormal frame field (2.2), we define the variational problem of elastic curves in two-dimensional lightlike cone $\mathbb{Q}^{2}$ as a critical point of the functional

$$
\phi(\gamma)=\int_{0}^{\ell}(-2 \kappa+\sigma) d s
$$

among the family of the spacelike curves

$$
\begin{aligned}
\gamma:[0, \ell] & \rightarrow \mathbb{Q}^{2} \\
s & \rightarrow \gamma(s)
\end{aligned}
$$

with the boundary conditions

$$
\left\|\gamma^{\prime}(s)\right\|=1, \quad \gamma(0)=p_{0}, \quad \gamma(\ell)=p_{\ell}, \quad \gamma^{\prime}(0)=V_{0}, \quad \gamma^{\prime}(\ell)=V_{\ell},
$$

where $V_{0} \in T_{p_{0}} \mathbb{Q}^{2}, V_{\ell} \in T_{p_{\ell}} \mathbb{Q}^{2}, \sigma \in \mathbb{R}$ is a fixed. Also $\ell$ is variable and $\kappa$ is the cone curvature function.

Let

$$
\begin{aligned}
\gamma:[0, \ell] & \rightarrow \mathbb{Q}^{2} \\
s & \rightarrow \gamma(s)=\left(\gamma_{1}(s), \gamma_{2}(s), \gamma_{3}(s)\right)
\end{aligned}
$$


be a curve parametrized by arc length $s, 0 \leqslant s \leqslant \ell$, with the cone curvature function $\kappa$ in the lightlike cone $\mathbb{Q}^{2}$. Then, the functional (3.1) write as the follow

$$
\int_{0}^{\ell}\left(<T^{\prime}(s), T^{\prime}(s)>+\sigma\right) d s
$$

under the constraints

$$
<T(s), T(s)>=1, \quad<\gamma(s), \gamma(s)>=0
$$

and

$$
\gamma^{\prime}(s)=T(s) .
$$

In order to find out the differential equations giving the critical points of the functional (3.2), we can carry out the Euler-Lagrange equations

to the functional

$$
\begin{aligned}
& \frac{\partial F}{\partial \gamma}-\frac{d}{d s}\left(\frac{\partial F}{\partial \gamma^{\prime}}\right)=0, \\
& \frac{\partial F}{\partial T}-\frac{d}{d s}\left(\frac{\partial F}{\partial T^{\prime}}\right)=0
\end{aligned}
$$

$$
\begin{aligned}
F & =<T^{\prime}(s), T^{\prime}(s)>+\sigma+\lambda(<T(s), T(s)>-1)+\mu(<\gamma(s), \gamma(s)>) \\
& +2<\Lambda, \gamma^{\prime}(s)-T(s)>
\end{aligned}
$$

where $\lambda$ and $\mu$ are scalars and $\Lambda=\left(\Lambda_{1}, \Lambda_{2}, \Lambda_{3}\right)$ is a vector in $E_{1}^{3}$ (for calculus of variations see [15]). So, we obtain from the Euler-Lagrange equations

$$
\mu\left(\gamma_{1}, \gamma_{2},-\gamma_{3}\right)-\left(\Lambda_{1}^{\prime}, \Lambda_{2}^{\prime},-\Lambda_{3}^{\prime}\right)=0
$$

and

$$
\lambda\left(t_{1}, t_{2},-t_{3}\right)-\left(t_{1}^{\prime \prime}, t_{2}^{\prime \prime},-t_{3}^{\prime \prime}\right)=\left(\Lambda_{1}, \Lambda_{2},-\Lambda_{3}\right),
$$

respectively, $[4,11,16]$. If we take first derivative of $(3.5)$ with respect to $s$ and combine with (3.4), we obtain

$$
\lambda^{\prime}\left(t_{1}, t_{2},-t_{3}\right)+\lambda\left(t_{1}^{\prime}, t_{2}^{\prime},-t_{3}^{\prime}\right)-\left(t_{1}^{\prime \prime \prime}, t_{2}^{\prime \prime \prime},-t_{3}^{\prime \prime \prime}\right)=\mu\left(\gamma_{1}, \gamma_{2},-\gamma_{3}\right) .
$$

By using the equations (2.2), we have some derivatives of $T$;

$$
\begin{gathered}
T^{\prime}(s)=\kappa(s) \gamma(s)-N(s), \\
T^{\prime \prime}(s)=\kappa^{\prime}(s) \gamma(s)+2 \kappa(s) T(s)
\end{gathered}
$$

and

$$
T^{\prime \prime \prime}(s)=\left(\kappa^{\prime \prime}(s)+2 \kappa^{2}(s)\right) \gamma(s)+3 \kappa^{\prime}(s) T(s)-2 \kappa(s) N(s) .
$$

Substituting these derivatives in (3.6), we have the equilibrium equation as the following;

$$
\left(\kappa^{\prime \prime}+2 \kappa^{2}-\lambda \kappa+\mu\right) \gamma+\left(-\lambda^{\prime}+3 \kappa^{\prime}\right) T+(\lambda-2 \kappa) N=0 .
$$

From the equation (3.7) and (2.1), we obtain

$$
\begin{gathered}
\kappa^{\prime \prime}+2 \kappa^{2}-\lambda \kappa+\mu=0, \\
-\lambda^{\prime}+3 \kappa^{\prime}=0
\end{gathered}
$$

and

$$
\lambda-2 \kappa=0 .
$$


Computing $\kappa$ from (3.8), (3.9) and (3.10), we get

$$
\kappa=c, \quad c=\text { constant } .
$$

Substituting (3.11) in (3.8), we obtain $\mu=0$. To specify the constant $c$ with respect to the tension parameter $\sigma$, we consider the boundary condition

$$
F(\ell)-\sum_{i=1}^{3} \frac{\partial F}{\partial \gamma_{i}^{\prime}}(\ell) \gamma_{i}^{\prime}(\ell)-\sum_{i=1}^{3} \frac{\partial F}{\partial t_{i}^{\prime}}(\ell) t_{i}^{\prime}(\ell)=0
$$

for the extremal $\gamma$. This condition is implied by the fact that the total length $\ell$ of the curve is a variable in the variation [2]. Thus, we have

$$
-2 \kappa(\ell)+\sigma-2<\Lambda(\ell), \gamma^{\prime}(\ell)>-2<T^{\prime}(\ell), T^{\prime}(\ell)>=0 .
$$

Substituting $\Lambda$, the components of $\Lambda$ in (3.5) into the inner product $<\Lambda(\ell), \gamma(\ell)>$ yields

$$
<\Lambda(\ell), \gamma(\ell)>=0 .
$$

From (3.11) and (3.12) one observes easily

$$
c=-\frac{\sigma}{2} .
$$

Therefore, we can give the following theorem which determine spacelike elastic curves in the lightlike cone $\mathbb{Q}^{2}$.

Theorem 3.1. $\gamma$ is a spacelike elastic curve in a two-dimensional lightlike cone $\mathbb{Q}^{2}$ if and only if the cone curvature of spacelike curve $\gamma$ is a constant which is equal negative half of the tension parameter.

In [7], authors gave a classification of the curves with constant cone curvature in a two-dimensional lightlike cone $\mathbb{Q}^{2}$. By using this classification, we can give the following corollary.

Corollary 3.1. If $\gamma(s)$ is spacelike elastic curve with arc length parameter $s$ in a two-dimensional lightlike cone $\mathbb{Q}^{2}$, then $\gamma(s)$ can be written as the following:

i) For $c_{1}, c_{2}, c_{3} \in \mathbb{R}_{1}^{3}$ and $\sigma=0$, the curve

$$
\gamma(s)=c_{1} s^{2}+c_{2} s+c_{3}
$$

is a parabola (Figure 2). Thus $\gamma$ is a free elastic curve.

ii) For $c_{1}, c_{2}, c_{3} \in \mathbb{R}_{1}^{3}$ and $\sigma<0$, the curve

$$
\gamma(s)=c_{1} \sinh (\sqrt{-\sigma}) s+c_{2} \cosh (\sqrt{-\sigma}) s+c_{3}
$$

is a hyperbola (Figure 3,4 ),

iii) For $c_{1}, c_{2}, c_{3} \in \mathbb{R}_{1}^{3}$ and $\sigma>0$, the curve

$$
\gamma(s)=c_{1} \sin (\sqrt{\sigma}) s+c_{2} \cos (\sqrt{\sigma}) s+c_{3}
$$

is an ellipse (Figure 5). 


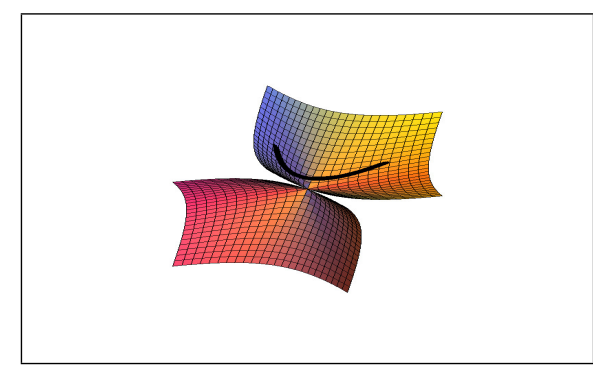

Figure $2 . \quad c_{1}=(1.6,0,0), c_{2}=(0,3.3,0), c_{3}=(0,0,3.1)$

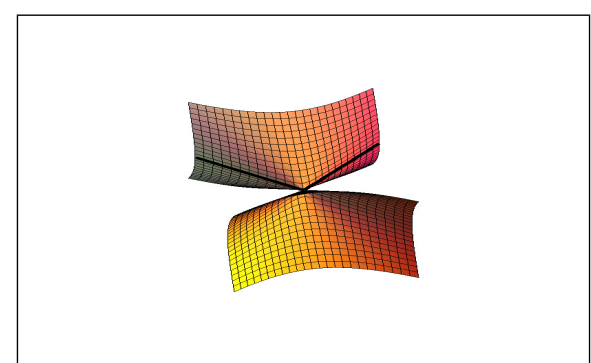

FiguRE 3. $\quad c_{1}=(0.055,0,0), c_{2}=(0,0.055,0), c_{3}=(0,0,0.055)$

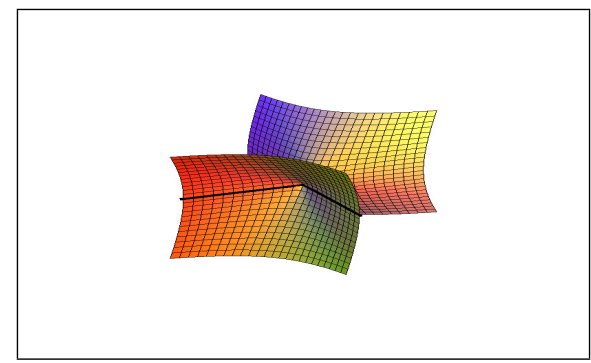

Figure 4. $c_{1}=(-0.065,0,0), c_{2}=(0,-0.065,0), c_{3}=(0,0,-0.065)$

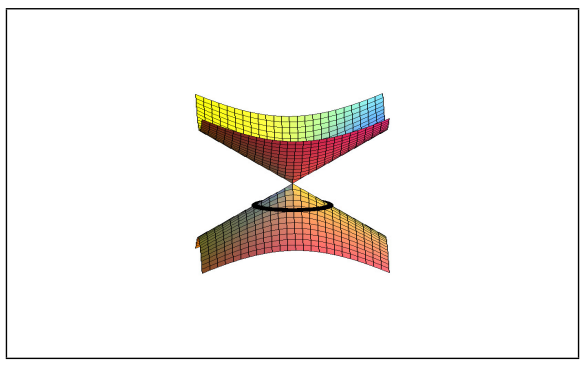

Figure 5. $c_{1}=(4,0,0), c_{2}=(0,4,0), c_{3}=(0,0,4)$ 


\section{REFERENCES}

[1] Barros, M., Ferrndez, A., Lucas, P. and Meroo, M.A., Willmore Tori and Willmore-Chen Submanifolds in Pseudo-Riemannian Spaces,Journal of Geometry and Physics, 28(1998), 45-66.

[2] Bolza, O., Vorlesungen Ueber Variationsrechnung, Koehler und Amelang, Leipzig, 1949.

[3] Brunnett, G., A New Characterization of Plane Elastica, Mathematical Methods in Computer Aided Geometric Design II (eds.) Tom Lyche and Larry L. Schumaker (Academic Press, Inc., 1992), 43-56.

[4] Brunnett, G. and Crouch, P.E., Elastic Curves on the Sphere, Naval Postgraduate School, Monterey, California, 1993.

[5] Huang, R., A Note on the p-elastica in a Constant Sectional Curvature Manifold, Journal of Geometry and Physics, 49(2004), 343-349.

[6] Langer, J. and Singer, D., The Total Squared Curvature of Closed Curves, Journal of Differential Geometry, 20(1984), 1-22.

[7] Liu, H., Curves in the Lightlike Cone, Beitrge zur Algebra und Geometrie Contributions to Algebra and Geometry, 44(2004), no. 1, 291-303.

[8] Liu, H., Meng, Q., Representation Formulas of Curves in a two and threedimensional Lightlike Cone, Results in Mathematics, 59(2011), 437-451.

[9] Lopez, R., Differential Geometry of Curves and Surfaces in Lorentz- Minkowski Space, International Electronic Journal of Geometry, 7(2014), no.1, 44-107.

[10] O'Neill, B., Semi-Riemannian Geometry with Applications to Relativity, Academic Pres., New York, 1993.

[11] Oral, M., Elastic Curves on Hyperquadrics in Minkowski 3-space, Master Thesis, Sleyman Demirel University, Graduate School of Natural and Applied Sciences, 2010, 40p.

[12] Ozkan, G., Elastic Strips in Minkowski 3-space, Doctoral Thesis, Sleyman Demirel University, Graduate School of Natural and Applied Sciences, 2014, 93p.

[13] Singer, D. Lectures on elastic curves and rods, AIP Conf. Proc. 1002, Amer. Inst. Phys., Melville. New York, 2008.

[14] Steinberg, D., H., Elastic Curves in Hyperbolic Space, Doctoral Thesis, Case Western Reserve University, UMI Microform 9607925, 1995, 72p.

[15] Weinstock, R., Calculus of Variations, McGraw-Hill Book Company, USA, 1952, 326p.

[16] Yucesan, A. and Oral, M., Elastica on 2-dimensional anti-De Sitter space, International Journal of Geometric Methods in Modern Physics, 8(2011), no.1, 107-113. 